\title{
Investigation on the Current Nonuniformity in Current-Mode TFT Active-Matrix Display Pixel Circuitry
}

\author{
Xiaojun Guo, Student Member, IEEE and S. R. P. Silva
}

\begin{abstract}
Detailed analysis of the mechanisms that cause deviations of the emission current from the input data current in polycrystalline silicon (poly-Si) thin-film transistor (TFT) currentcopier active matrix carbon nanotube field emission display (FED) pixel circuits is presented. These effects make the modulated emission current sensitive to the process variations of the circuit elements. Monte Carlo circuit analysis with a Gaussian statistical distribution of all related process parameters in the pixel circuit shows the emission current nonuniformity, and therefore illustrates the importance of improving the poly-Si TFT process for the design of high-resolution and high-brightness current-mode active matrix addressed displays. The analysis is also suitable for design and optimization of other reported current-mode active matrix addressed FED or organic light-emitting displays.
\end{abstract}

Index Terms-Active matrix, carbon nanotube (CNT), currentcopier, current-mode, field emission display (FED), flat-panel display, Monte Carlo, organic light-emitting display (OLED), polycrystalline silicon (poly-Si), thin film transistor (TFT).

\section{INTRODUCTION}

$\mathbf{F}$ IELD emission displays (FED) and organic light-emitting displays (OLEDs) are currently the two most promising display technologies that are likely to replace the dominant liquid crystal display in the future [1], [2]. In both technologies, the luminance of the lighting element is proportional to its current density, and therefore current-mode pixel driving approaches appear to be a natural solution for high quality FEDs or OLEDs. To date, current-copier and current-mirror type pixel circuitry based on amorphous silicon (a-Si) or polycrystalline silicon thin film transistor (poly-Si TFT) technologies have been proposed for the active matrix addressed OLEDs [3], [4]. Lin et al. [5] used a current-mirror-based switch-current memory pixel circuit for OLEDs to improve the accuracy and operation frequency, and Levy et al. [6] has already designed a CMOS subthreshold-voltage-scaling current-mirror driver for OLED-on-silicon to improve the bandwidth and settling behavior. Recently, the design of poly-Si TFT current-modulated FED pixels based on carbon nanotube (CNT) emitters was also reported [7]. These current-mode pixel drivers greatly improve the display uniformity, compared with the voltage-mode

Manuscript received April 19, 2005. This work was supported by the EPSRC Portfolio Partnership Award and the Overseas Research Scholarship. The review of this paper was arranged by Editor H.-S. Tae.

The authors are with the Nano-Electronics Center, Advanced Technology Institute, University of Surrey, Surrey GU2 7XH, U.K. (e-mail: x.guo@surrey.ac.uk)

Digital Object Identifier 10.1109/TED.2005.857936
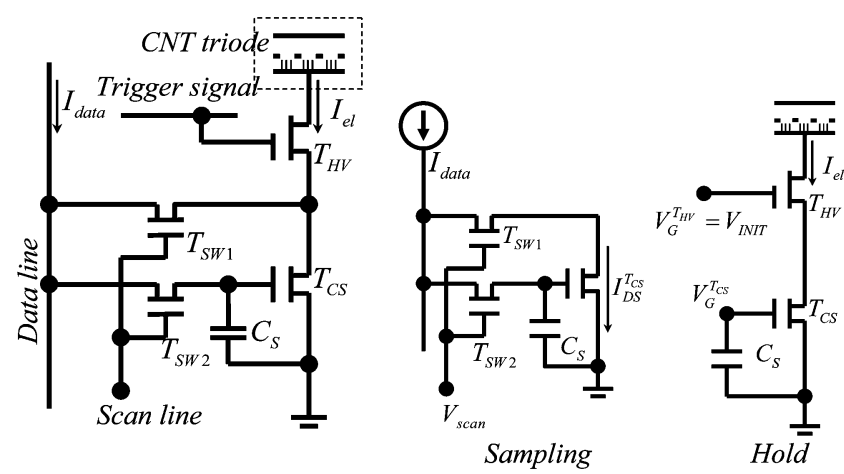

Fig. 1. Current-copier carbon nanotube (CNT) based FED pixel circuit and its two operation phase: sampling and hold. $I_{\mathrm{data}}$ is the input data current, and $I_{\mathrm{el}}$ is modulated emission current of the CNT field emission device.

pixel drivers [8], [9], as well as successfully achieving good gray-shade control by continuously modulating the current density of each pixel directly with the input data current.

For the current-copier CNT-FED pixel circuit, as shown in Fig. 1, the operation can be divided into two phases: sampling and hold. During the sampling phase, the video signal is converted to a current signal $I_{\text {data }}$, then sampled into the current sink transistor $T_{\mathrm{CS}}$ during row selection. During the hold phase, the current sink transistor $T_{\mathrm{CS}}$ reproduces and holds the current signal to drive the lighting element. Theoretically, the modulated emission current $I_{\mathrm{el}}$ is equal to the input data current $I_{\text {data }}$, independent of the process variations of the circuit components. However, there are usually some deviations of $I_{\mathrm{el}}$ from $I_{\text {data }}$ due to several undesired effects which are sensitive to the process variations of circuit components. Thus, the output linearity of the driver circuitry and the emission current uniformity may deteriorate.

In this paper, the mechanisms that cause the current deviations from ideal are carefully investigated for the first time. A modification of the pixel circuitry in Fig. 1, with an additional feedback capacitance between $T_{\mathrm{CS}}$ 's source and drain is proposed and analyzed. The Monte Carlo analysis, taking account of the process variations, predicts the performance of the current-copier pixel circuits in a true poly-Si TFT process. The circuit simulation in the work is based on the RPI Polysilicon TFT model using the Silvaco Smart-Spice circuit simulator [10]. The setup for the simulation can be seen in [7]. Although the study in this work is based on the current-copier FED pixel circuit, it is also very suitable for other current-mode active matrix display pixel drivers for FEDs and OLEDs. 


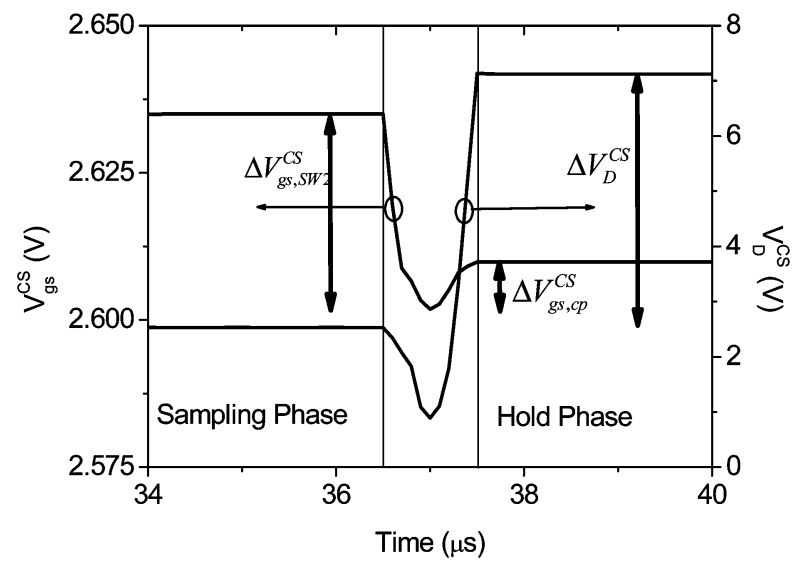

Fig. 2. Variation of the $T_{\mathrm{CS}} \mathrm{s}$ gate/source voltage $V_{\mathrm{gS}}^{\mathrm{CS}}$ and drain/source voltage $V_{D}^{\mathrm{CS}}$ when the pixel circuit in Fig. 1 shifts from sampling phase to hold phase: $\Delta V_{\mathrm{gs} \cdot \mathrm{SW} 2}^{\mathrm{CS}}$ is the falling part due to charge injection and clock feed-through effects on $T_{\mathrm{SW} 2}, \Delta V_{\mathrm{gs} \text { cp }}^{\mathrm{CS}}$ is the rising part due to $T_{\mathrm{CS}} \mathrm{S}$ drain-to-gate coupling, and $\Delta V_{D}^{\mathrm{CS}}$ is $T_{\mathrm{CS}} \mathrm{s}$ drain/source voltage variation.

\section{ANALYSIS OF THE CURRENT ERRORS}

For the current-copier FED pixel shown in Fig. 1, in a realistic case, the finally modulated emission current is given by [11]

$$
I_{\mathrm{el}}=\left(\sqrt{I_{\mathrm{data}}}-\sqrt{\beta_{\mathrm{CS}}} \cdot \Delta V_{\mathrm{gs}}^{\mathrm{CS}}\right)^{2}+\lambda \cdot \Delta V_{D}^{\mathrm{CS}}
$$

where $\beta_{\mathrm{CS}}=(1 / 2) \cdot(W / L) \mu_{F E} \cdot C_{\mathrm{oX}}$ is the transconductance factor of $T_{\mathrm{CS}}, \Delta V_{\mathrm{gs}}^{\mathrm{CS}}$ and $\Delta V_{D}^{\mathrm{CS}}$ are the gate stored voltage and the drain voltage shift which occurs when the pixel operation changes from the sampling phase to the hold phase, and $\lambda$ is a constant associated with the channel length modulation and the kink effects of the poly-Si TFTs.

Equation (1) shows that $\Delta V_{\mathrm{gs}}^{\mathrm{CS}}$ and $\Delta V_{D}^{\mathrm{CS}}$ may cause deviation of $I_{\mathrm{el}}$ from $I_{\mathrm{data}}$. As illustrated in Fig. 2, $\Delta V_{\mathrm{gs}}^{\mathrm{CS}}$ is composed of two parts: the falling part $\Delta V_{\mathrm{gs} \cdot \mathrm{SW} 2}^{\mathrm{CS}}$ due to the charge injection and clock feed-through effects on $T_{\mathrm{SW} 2}$ and the rising part $\Delta V_{\mathrm{gs} \cdot \mathrm{CP}}^{\mathrm{CS}}$ due to $T_{\mathrm{CS}} \mathrm{s}$ drain-to-gate coupling. The following discussions will analyze the two parts of $\Delta V_{\mathrm{gs}}^{\mathrm{CS}}$ and present the mechanisms that produce $\Delta V_{\mathrm{gs}}^{\mathrm{CS}}, \Delta V_{D}^{\mathrm{CS}}$ as well as the relations between $I_{\mathrm{el}}, \Delta V_{\mathrm{gs}}^{\mathrm{CS}}, \Delta V_{D}^{\mathrm{CS}}$ and the circuit components.

\section{A. Impacts of $T_{\mathrm{Sw} 2}$ S Switching Effects}

The main factor that induces the sampled $V_{\mathrm{gs}}^{\mathrm{cs}}$ error is the charge injection and clock feedthrough effects of $T_{\mathrm{SW} 2}$. In Fig. 1, as $V_{\text {scan }}$ begins to fall to turn off the switch, mobile charges exit through both the drain end and the source end of $T_{\mathrm{SW} 2}$. The charges transferred to the $C_{S}$ cause a reduction to the sampled voltage, which can be expressed as [12]

$$
\Delta V_{c h}=\frac{p W L C_{\mathrm{ox}}\left(V_{\mathrm{scan}}-V_{T}^{\mathrm{SW} 2}-\eta V_{\mathrm{gs}}^{\mathrm{CS}}\right)}{C_{S}+C_{\mathrm{gs}}^{\mathrm{CS}}}
$$

where $V_{T}^{\mathrm{SW} 2}$ is $T_{\mathrm{SW} 2} \mathrm{~S}$ threshold voltage, $p$ denotes the fraction of the channel charge that flows into $C_{S}$, depending on the voltage swing and falling rate of $V_{\text {scan }}$. A higher swing or quicker falling rate of $V_{\mathrm{scan}}$ will cause larger $p . \eta V_{\mathrm{gs}}^{\mathrm{CS}}$ represents the average channel potential, which is dependent on the input data current.

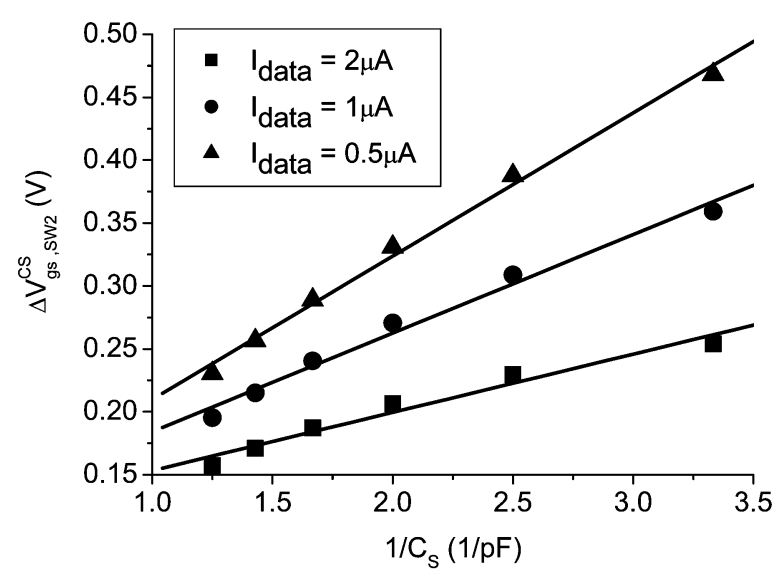

(a)

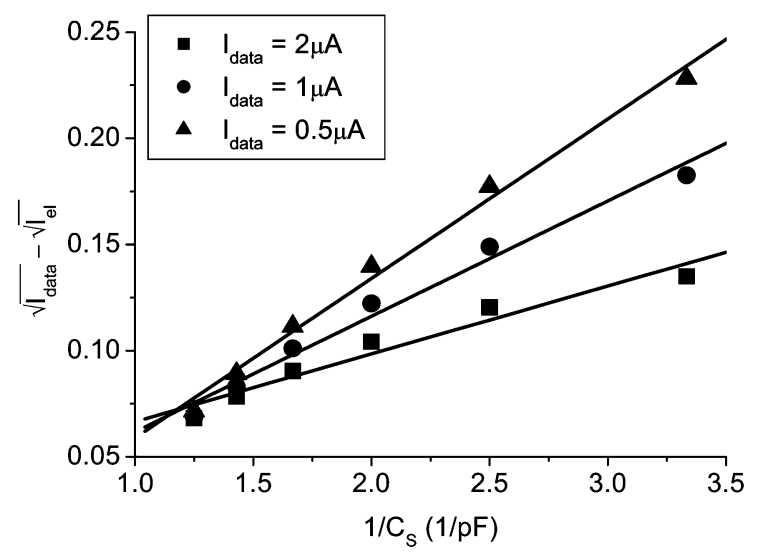

(b)

Fig. 3. Impacts of $C s$ with different $I_{\text {data }}$. (a) Dependence of $\Delta V_{\mathrm{gs}, S W 2}^{\mathrm{CS}}$, which is illustrated in Fig. 2, on $C_{\mathrm{CS}}$. (b) Dependence of $\sqrt{I_{\mathrm{data}}}-\sqrt[3]{I_{\mathrm{el}}}$ on $C_{S}$.

At the same time, when $V_{\text {scan }}$ switches from high to low, the voltage difference in $V_{\text {scan }}$ switching may be coupled into the sampled point with the clock feed-through effect, and give rise to $\Delta V_{c l}$, which is given by

$$
\Delta V_{c l}=\frac{V_{\mathrm{scan}} \cdot C_{\mathrm{gs}}^{\mathrm{SW} 2}}{C_{\mathrm{gs}}^{\mathrm{SW} 2}+C_{S}} .
$$

For the self-aligned TFT technology, $C_{\mathrm{gs}}^{\mathrm{SW} 2} \approx(1 / 2)(W$. $L)_{\mathrm{SW} 2} \cdot C_{\mathrm{ox}}^{\mathrm{SW} 2}$ is much smaller than $C_{S}$. Thus, the total amount that the settling voltage $V_{\mathrm{gS}}^{\mathrm{CS}}$ is pulled down can be approximated as

$$
\begin{aligned}
\Delta V_{\mathrm{gs}, \mathrm{SW} 2}^{\mathrm{CS}}= & \Delta V_{c h}+\Delta V_{c l} \\
= & \frac{(W \cdot L)_{\mathrm{SW} 2} C_{\mathrm{ox}}^{\mathrm{SW} 2}}{C_{S}} \\
& \times\left[p\left(V_{\mathrm{scan}}-V_{T}^{\mathrm{SW} 2}-\eta V_{\mathrm{gs}}^{\mathrm{CS}}\right)+\frac{1}{2} V_{\mathrm{scan}}\right] .
\end{aligned}
$$

Figs. 3 and 4 show the dependence of $\Delta V_{\mathrm{gs}, \mathrm{SW} 2}^{\mathrm{CS}}, I_{\mathrm{el}}$ on $C_{S}$ and the dimensions of $T_{\mathrm{SW} 2}$, respectively. They fit the linear relations of $\Delta V_{\mathrm{gs}, \mathrm{SW} 2}^{\mathrm{CS}} \sim 1 / C_{s},\left(\sqrt{I_{\mathrm{data}}}-\sqrt{I_{\mathrm{el}}}\right) \sim 1 / C_{s}$, $\Delta V_{\mathrm{gs}, \mathrm{SW} 2}^{\mathrm{CS}} \sim W_{\mathrm{CS}}$, and $\left(\sqrt{I_{\mathrm{data}}}-\sqrt{I_{\mathrm{el}}}\right) \sim W_{\mathrm{CS}}$, derived from (1) and (4). The decrease of $\Delta V_{\mathrm{gs}, \mathrm{SW} 2}^{\mathrm{CS}}$ with increased 


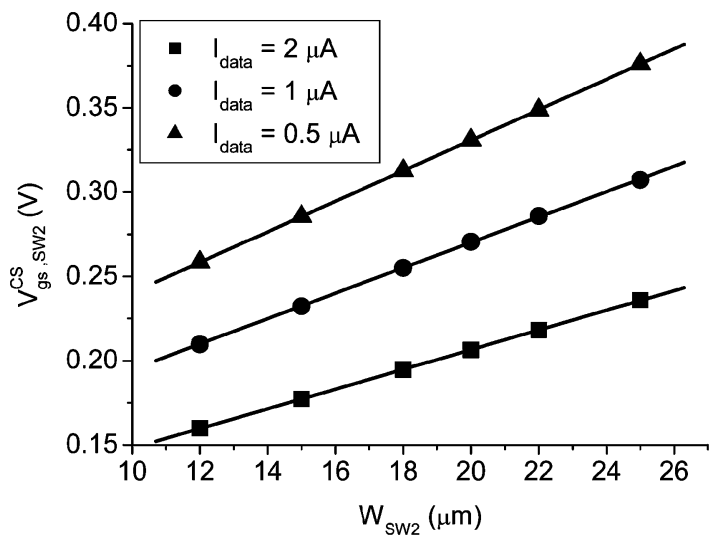

(a)

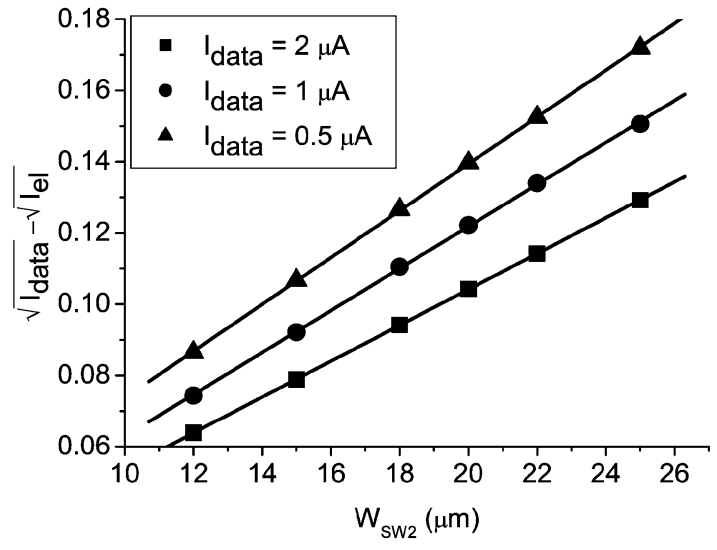

(b)

Fig. 4. Impacts of $T_{\mathrm{SW} 2}$ 's width $W_{\mathrm{SW} 2}$ with different $I_{\text {data }}$. (a)Dependence of $T_{\mathrm{CS}}$ 's gate/source voltage change $\Delta V_{\mathrm{gs}, \mathrm{SW} 2}^{\mathrm{CS}}$, which is illustrated in Fig. 2, on $W_{\mathrm{SW} 2}$. (b) Dependence of $\sqrt{I_{\mathrm{data}}}-\sqrt{I_{\mathrm{el}}}$ on $W_{\mathrm{SW} 2}$.

$I_{\text {data }}$ shown in Figs. 3 and 4 also agrees with (4), since a larger $I_{\text {data }}$ brings a larger $\eta V_{\mathrm{gs}}^{\mathrm{CS}}$, which causes smaller $\Delta V_{\mathrm{gs}, \mathrm{SW} 2}^{\mathrm{CS}}$ according to (4).

Based on the above arguments, $\Delta V_{\mathrm{gs}, \mathrm{SW} 2}^{\mathrm{CS}}$ can be minimized by using the largest $C_{s}$ possible, using minimum-geometry $T_{\text {SW2 }}$, keeping the $V_{\text {scan }}$ swings as small as possible and slowing the falling rate. However, decreasing $T_{\mathrm{SW} 2} \mathrm{~s}$ length will cause larger leakage current in the hold phase; increasing $C_{s}$ and decreasing $T_{\mathrm{SW} 2} \mathrm{~S}$ width will prolong the required settling time; lowering the $V_{\text {scan }}$ swing will increase $T_{\mathrm{SW} 1}, T_{\mathrm{SW} 2} \mathrm{~s}$ ON resistance and thereafter the settling time; finally, the limited pixel select-on time makes it difficult to slow $V_{\text {scan }} \mathrm{s}$ falling rate too much. That implies some compromises are necessary to be considered in the design to achieve the best performance, and $\Delta V_{\mathrm{gs}, \mathrm{SW} 2}^{\mathrm{CS}}$ is difficult to be completely eliminated.

\section{B. $T_{\mathrm{CS}}$ Drain-to-Gate Coupling}

Another effect that contributes to the sampled $V_{\mathrm{gs}}^{\mathrm{cs}}$ error is the drain-to-gate coupling of $T_{\mathrm{CS}}$ through the drain/gate capacitance $C_{\mathrm{gd}}$. When the circuit switches from the sampling phase to the hold phase, the increase of $V_{D}^{\mathrm{CS}}$ may be coupled to the gate with the parasitic drain/gate capacitance $C_{\mathrm{gd}}$, and increase

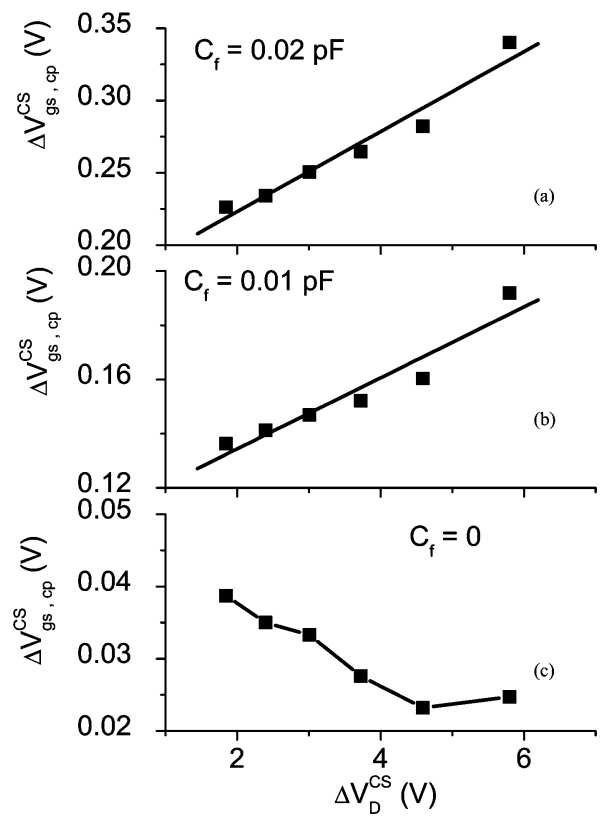

Fig. 5. Dependence of $\Delta V_{\mathrm{gss}}^{\mathrm{CS}}$, which is illustrated in Fig. 2, on $\Delta V_{D}^{\mathrm{CS}}$, with different additional feed-back capacitances $C_{f}$ between $T_{\mathrm{CS}} \mathrm{s}$ drain and gate. (a) $C_{f}=0.02 \mathrm{pF}$. (b) $C_{f}=0.01 \mathrm{pF}$. (c) $C_{f}=0$.

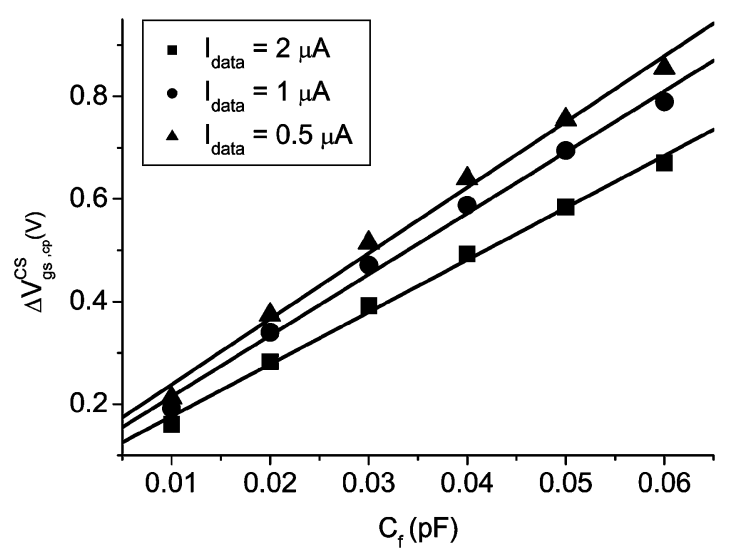

Fig. 6. Dependence of $\Delta V_{\mathrm{gs}, \mathrm{cp}}^{\mathrm{CS}}$, which is illustrated in Fig. 2, on the additional feed-back capacitance $C_{f}$ between $T_{\mathrm{CS}} \mathrm{s}$ drain and gate.

the $V_{\mathrm{gs}}^{\mathrm{CS}}$ with a certain amount $\Delta V_{\mathrm{gs}, \mathrm{cp}}^{\mathrm{CS}}$, which can be expressed as [12]

$$
\Delta V_{\mathrm{gs}, \mathrm{cp}}^{\mathrm{CS}}=\frac{\Delta V_{D}^{\mathrm{CS}} \cdot C_{\mathrm{gd}}}{C_{S}+C_{\mathrm{gd}}}
$$

For the self-aligned TFT technology, the $C_{\mathrm{gd}}$ is mainly composed of the gate/channel capacitance, which depends on the operation state. With the increased drain voltage, the depletion region near the drain increases and $C_{\mathrm{gd}}$ decreases. Thus, $\Delta V_{\mathrm{gs}, \mathrm{cp}}^{\mathrm{CS}}$ may decrease with increased $V_{D}^{\mathrm{CS}}$ in this case, as shown in Fig. 5(c). If adding a larger feedback capacitance $C_{f}$ between the gate and drain of $T_{\mathrm{CS}}, C_{\mathrm{gd}} \approx C_{f}$, and in Fig. 5(a) and (b), $\Delta V_{\mathrm{gs}, \mathrm{cp}}^{\mathrm{CS}}$ has a linear rise while increasing $V_{D}^{\mathrm{CS}}$, which accords with (5). The linear relation between $\Delta V_{\mathrm{gs}, \mathrm{cp}}^{\mathrm{CS}}$ and $C_{\mathrm{gd}}$ from (5) also fits the simulation results in Fig. 6. 


\section{The Impacts of $T_{\mathrm{SW} 1}$ ON-Resistance}

When the data current is sampled into the current sink transistor $T_{\mathrm{CS}}$, the nonzero resistance of $T_{\mathrm{SW} 1}$ causes a voltage drop $\Delta V_{\mathrm{SW} 1}$ on the switch. So there is

$$
V_{\mathrm{DS}}^{\mathrm{CS}}=V_{\mathrm{GS}}^{\mathrm{CS}}-\Delta V_{\mathrm{SW} 1}
$$

where

$$
\Delta V_{\mathrm{SW} 1}=\frac{I_{\mathrm{data}}}{\mu_{\mathrm{SW} 1} \cdot\left(\frac{W}{L}\right)_{T_{\mathrm{SW} 1}} \cdot\left(V_{\mathrm{GS}}^{S W 1}-V_{T}^{S W 1}\right)}
$$

and

$$
V_{\mathrm{GS}}^{T_{\mathrm{SW} 1}}=V_{\mathrm{scan}}-I_{\mathrm{data}} \cdot r_{o}^{\mathrm{CS}} .
$$

$\Delta V_{\mathrm{SW} 1}$ must be kept small enough $\left(\Delta V_{\mathrm{SW} 1}<V_{T}^{\mathrm{CS}}\right)$, otherwise $T_{\mathrm{CS}}$ will be drawn into the triode operation region and bring a large sampled error of $V_{\mathrm{gs}}^{\mathrm{CS}}$ for reproducing the sampled current during hold phase. In the final design, $\Delta V_{\mathrm{SW} 1}$ can easily be made small enough. But its being sensitive to the process parameter variations of both $T_{\mathrm{CS}}$ and $T_{\mathrm{SW} 1}$, as seen from (6)-(8), may give rise to some variations of sampled $V_{\mathrm{gs}}^{\mathrm{CS}}$ and $V_{D}^{\mathrm{CS}}$. The larger $\Delta V_{\mathrm{SW} 1}$ will cause smaller $V_{D}^{\mathrm{CS}}$, and for a given input data current, the smaller $V_{D}^{\mathrm{CS}}$ induces larger $V_{\mathrm{gs}}^{\mathrm{CS}}$ based on (9). Thereafter, a larger emission current will be reproduced during the hold phase.

$$
I_{\mathrm{data}}=g_{m}^{\mathrm{CS}} \cdot V_{\mathrm{gs}}^{\mathrm{CS}}+\lambda \cdot V_{D}^{\mathrm{CS}}
$$

Fig. 7 shows the linear relation between $\Delta V_{\mathrm{SW} 1}$ and the $L / W$ of $T_{\mathrm{SW} 1}$, and the increased $\Delta I_{\mathrm{err}}=I_{\mathrm{el}}-I_{\text {data }}$ with increasing $L / W$ of $T_{\mathrm{SW} 1}$, which agrees with the above arguments very well. From (9), increasing the conductance $g_{m}^{\mathrm{CS}}$ of $T_{\mathrm{CS}}$ may cause a decrease of $V_{\mathrm{gs}}^{\mathrm{CS}}$ But, the increase of the conductance $g_{m}^{\mathrm{CS}}$ will also increase $\Delta V_{\mathrm{SW} 1}$, reduce $V_{D}^{\mathrm{CS}}$, and finally lower the $V_{\mathrm{gs}}^{\mathrm{CS}}$. Therefore, an increase of $\Delta I_{\text {err }}$ with increased carrier effective mobility of $T_{\mathrm{CS}}$ and $\mu_{\mathrm{CS}}$ can be found in Fig. 8 .

\section{Current-Copier PiXel Circuit With A FEEDBACK CAPACITANCE}

The main factor that induces the deviation of $I_{\mathrm{el}}$ from $I_{\mathrm{data}}$ is charge injection and clock through effects of $T_{\mathrm{SW} 2}$, and is possibly partially cancelled by adding a dummy transistor [13] or using a CMOS transmission gate instead of the n-TFT switch [14]. However, they both increase the pixel complexity with an additional TFT and input pulse signal. From Fig. 2, it is seen that the $\Delta V_{\mathrm{gs}, \mathrm{cp}}^{\mathrm{CS}}$ may counteract $\Delta V_{\mathrm{gs}, \mathrm{SW} 2}^{\mathrm{CS}}$. In fact, as illustrated in (5), Figs. 6 and 7, since the curve of $\Delta V_{d}^{\mathrm{CS}} \sim I_{\text {data }}$ has a similar change with that of $\Delta V_{\mathrm{gs}, \mathrm{SW} 2}^{\mathrm{CS}} \sim I_{\text {data }}$ (Fig. 9), choosing a suitable $C_{f}$ may produce a $\Delta V_{\mathrm{gs}, \mathrm{cp}}^{\mathrm{CS}}$ that can effectively reduce $\Delta V_{\mathrm{gs}, \mathrm{SW} 2}^{\mathrm{CS}}$ over the whole current range. Fig. 10 shows the improved linearity of the pixel circuit with adding a feedback capacitance $C_{f}$ between $T_{\mathrm{CS}}$ s gate and drain.

\section{CurRent Nonuniformity Due to Process VARIATIONS}

To predict the emission current nonuniformity of the pixel circuitry fabricated in a true poly-Si TFT foundry process, Monte
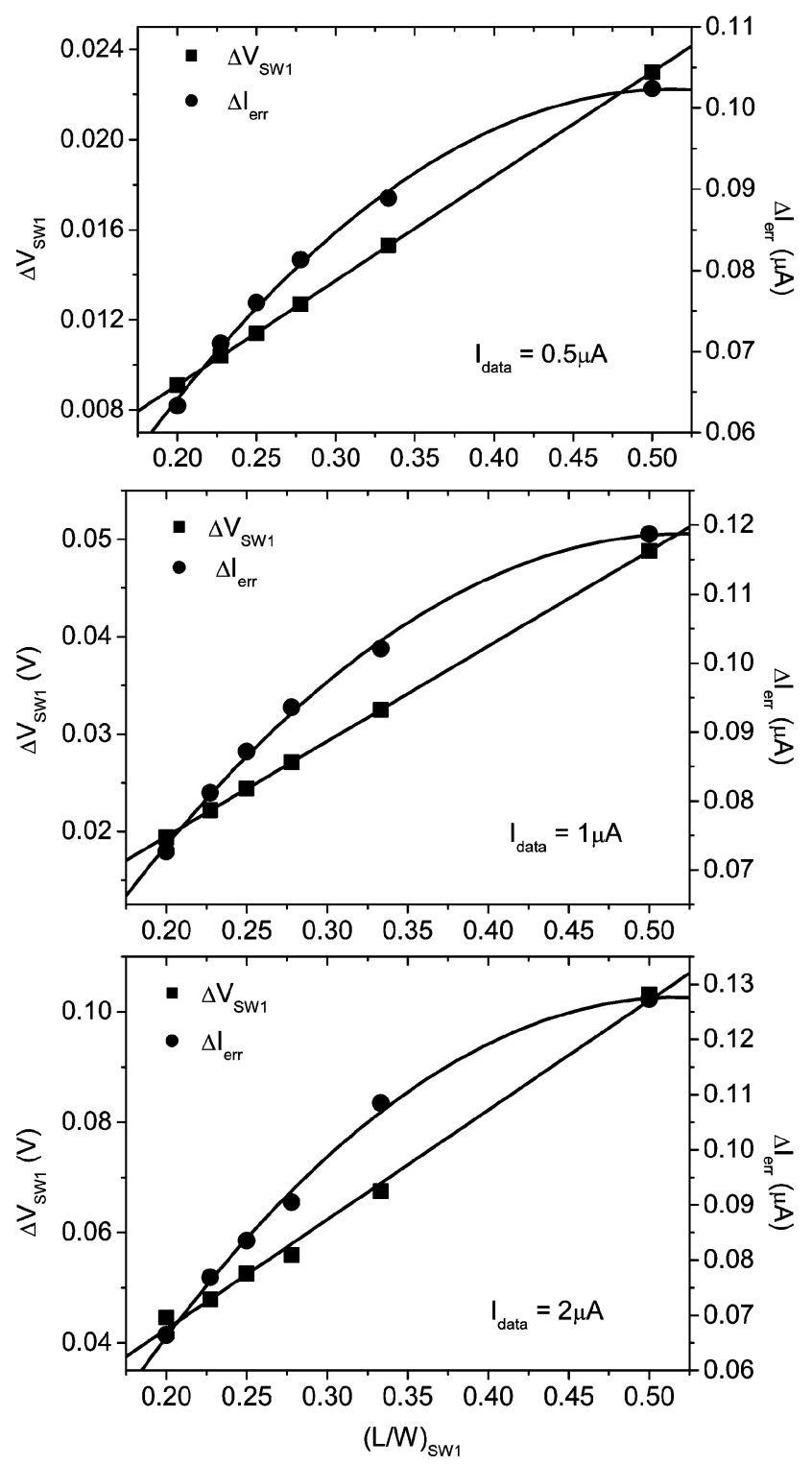

Fig. 7. Dependence of voltage drop on $T_{\mathrm{SW} 1} \Delta V_{\mathrm{SW} 1}$ and $\Delta I_{\mathrm{err}}=I_{\mathrm{el}}-I_{\text {data }}$ on $T_{\mathrm{SW} 1} \mathrm{~s}$ length-to-width ratio $(L / W)_{\mathrm{SW} 1}$.

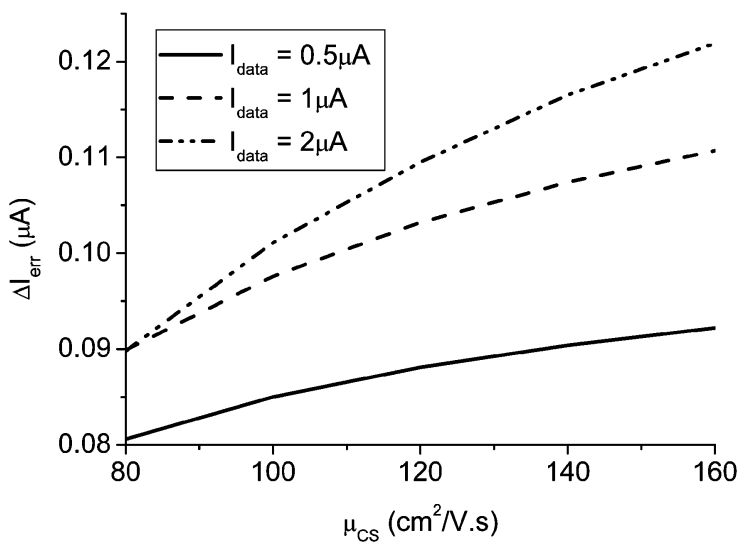

Fig. 8. Dependence $\Delta I_{\text {err }}=I_{\text {el }}-I_{\text {data }}$ on $T_{\mathrm{CS}}$ S effective carrier mobility $\mu_{\mathrm{CS}}$ of with different input data current $I_{\text {data }}$. 


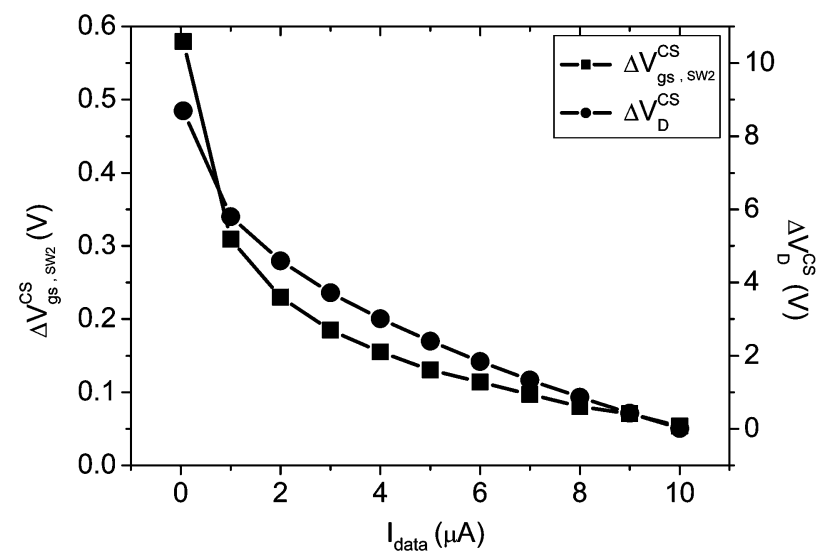

Fig. 9. Similar variations of $\Delta V_{\mathrm{gsSW} 2}^{\mathrm{CS}}$ and $\Delta V_{D}^{\mathrm{CS}}$ with $I_{\mathrm{data}}$ for the pixel circuit with an additional feed-back capacitance $C_{f}$ between $T_{\mathrm{CS}} \mathrm{s}$ drain and gate.

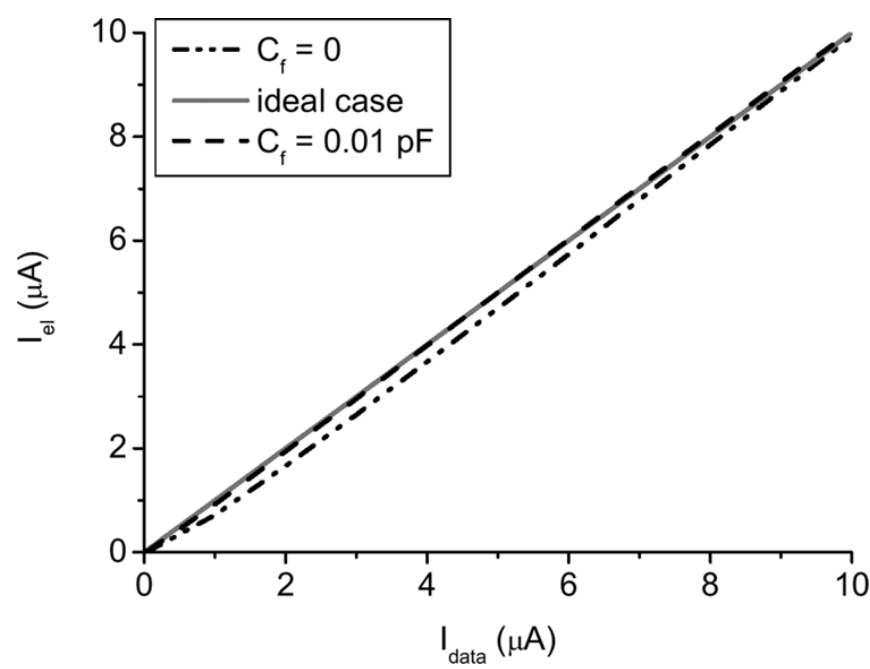

Fig. 10. Improved output linearity of the current-copier circuit with a feedback capacitance $C_{f}$, between the gate and drain of $T_{\mathrm{CS}}$.

Carlo analysis was performed with the Gaussian statistical distribution of all related process parameters in the circuit as shown in Table I. The nonuniform current-voltage characteristics and the standard deviation current errors of the triode field emitter model used for the simulation are shown in Fig. 11.

The distribution histogram of $I_{\mathrm{el}}$ obtained by Monte Carlo analysis in Fig. 12 shows the circuit with a feedback capacitance $C_{f}$, provides better current-copy accuracy, but it has a little worse uniformity due to the additional parameter $C_{f}$, which is also shown in Fig. 13. Although compared with the original current-voltage characteristics shown in Fig. 11, the standard deviation error for the emission current has been greatly reduced and therefore a much higher uniformity obtained, the increasing standard deviation of $I_{\mathrm{el}}$ with the increased $I_{\mathrm{data}}$ signifies the importance for improving the process for building high-resolution and high-brightness current-mode active matrix addressed displays. Fortunately, because the human eye is not sensitive to a smooth luminance variation in the long-range regime (a range of viewing angles from about $30^{\circ}$ to $60^{\circ}$ ) [15] and the TFT technology in a short-range regime can show good uniformity of the electrical characteristics, it is very promising to design the cur-

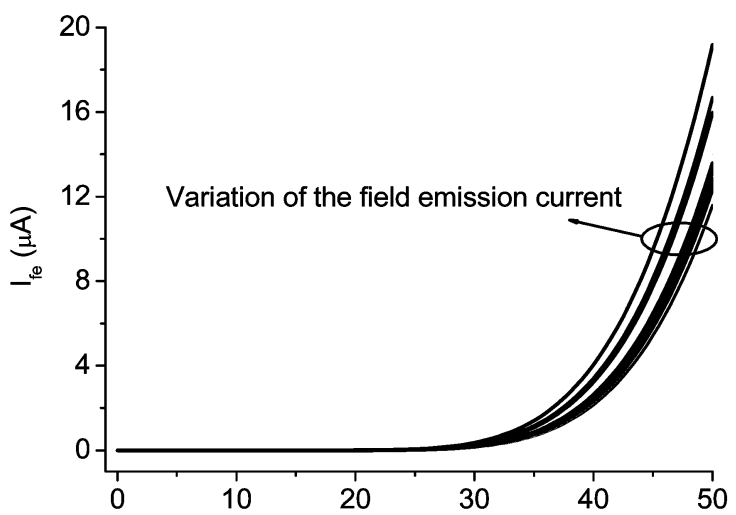

(a)

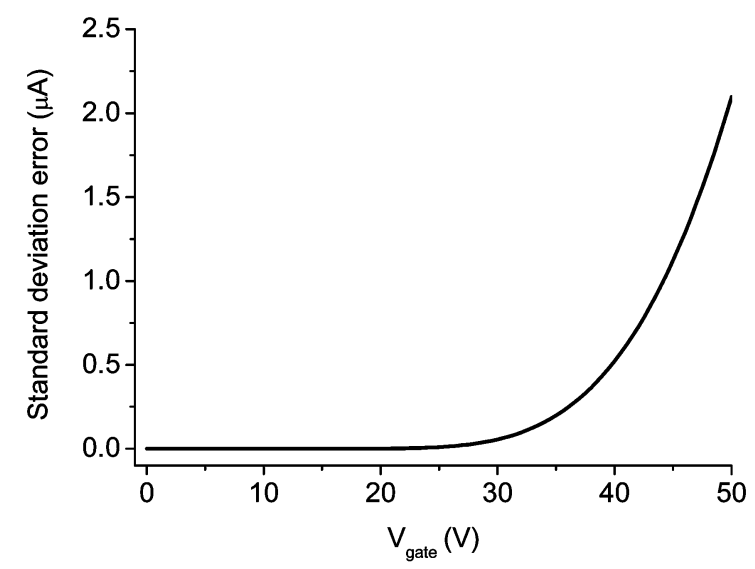

(b)

Fig. 11. (a) Current-voltage characteristics of the triode field emitter model for statistical simulation. (b) Standard deviation error of the field emission current with different gate voltages of the triode field emitter model.

TABLE I

Gauss Distribution of the Process Parameters FOR SPICE MONTE CARLO ANALYSIS

\begin{tabular}{l|l|l|l}
\hline Parameters & Normal value & $\begin{array}{l}\text { Related } \\
\text { variation }\end{array}$ & $\sigma$ \\
\hline$V_{T}\left(T_{C S, S W 1, S W 2}\right)$ & $1.5 \mathrm{~V}$ & $20 \%$ & 3 \\
\hline$V_{T}\left(T_{H V}\right)$ & $3.1 \mathrm{~V}$ & $20 \%$ & 3 \\
\hline$\mu\left(T_{C S, S W 1, S W 2}\right)$ & $130 \mathrm{~cm}^{2} / \mathrm{V} \cdot \mathrm{s}$ & $20 \%$ & 3 \\
\hline$\mu\left(T_{H V}\right)$ & $100 \mathrm{~cm}^{2} / \mathrm{V} \cdot \mathrm{s}$ & $20 \%$ & 3 \\
\hline kink effect factor & 19.3 & $15 \%$ & 3 \\
$\left(T_{C S, S W 1, S W 2}\right)$ & & $15 \%$ & 3 \\
\hline kink effect factor & 20.8 & $10 \%$ & 3 \\
\hline$\left(T_{H V}\right)$ & & $10 \%$ & 3 \\
\hline$W, L\left(T_{C S}\right)$ & $10 \mu \mathrm{m}$ & $10 \%$ & 3 \\
\hline$W\left(T_{S W 1, S W 2}\right)$ & $20 \mu \mathrm{m}$ & $10 \%$ & 3 \\
\hline$L\left(T_{S W 1, S W 2}\right)$ & $5 \mu \mathrm{m}$ & $10 \%$ & 3 \\
\hline$W\left(T_{H V}\right)$ & $15 \mu \mathrm{m}$ & $10 \%$ & 3 \\
\hline$L\left(T_{H V}\right)$ & $30 \mu \mathrm{m}$ & $10 \%$ & 3 \\
\hline$C_{S}$ & $0.4 \mathrm{pF}$ & & \\
\hline$C_{f}$ & $0.01 \mathrm{pF}$ &
\end{tabular}

rent-mode pixel circuit for produce FEDs or OLEDs with good enough luminance uniformity. 

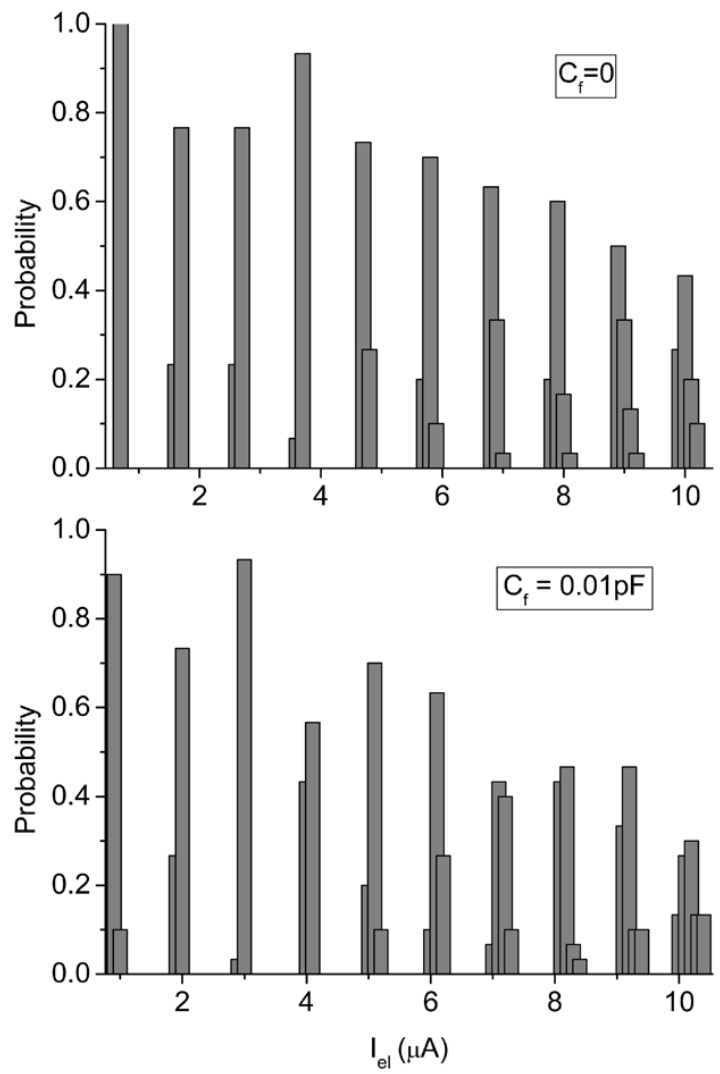

Fig. 12. $I_{\text {el }}$ distribution with different input $I_{\text {data }}$ obtained by Monte Carlo statistical analysis with the Gaussian distribution of process parameters shown in Table I.

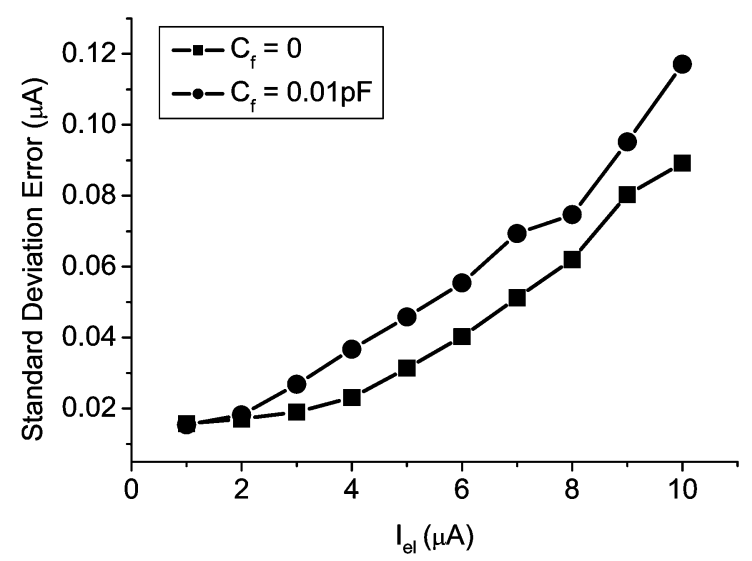

Fig. 13. Standard deviation of $I_{\mathrm{el}}$ obtained from the Monte Carlo analysis results.

\section{CONCLUSION}

The factors that contribute to the current nonuniformity in the poly-Si TFT current-copier FED pixel circuit are carefully investigated, including; 1) the charge injection and clock feedthough effects, 2) drain-to-gate coupling of the current sink TFT and, 3) the ON-resistance of the switch TFT. Detailed analysis of the modulated emission current varying with the circuit component parameters is very important for designing and optimizing the pixel circuit for high performance displays. It is also found that through adding a feedback capacitance, the output linearity of the circuit can be improved at some comprise of emission current uniformity. Monte Carlo analysis shows the nonuniformity of the current-copier circuit, and proves the importance of greatly improving the process to develop high-resolution and high-brightness active matrix addressed displays. The work in this paper is also useful to analyze and optimize other current-mode active matrix pixel drive circuits that have been reported.

\section{REFERENCES}

[1] S. Itoh and M. Tanaka, "Current status of field emission displays," Proc. IEEE, vol. 90, no. 4, pp. 514-520, Apr. 2002.

[2] H. Kanno, Y. Hamada, and H. Takahashi, "Development of OLED with high stability and luminance efficiency by co-doping methods for full color displays," IEEE J. Sel. Topics Quantum Electron., vol. 10, no. 1, pp. 30-36, Jan./Feb. 2004.

[3] Y. Hong, J. Nahm, and J. Kanicki, "100 dpi 4-a-Si:H TFTs active-matrix organic polymer light-emitting display," IEEE J. Sel. Topics Quantum Electron., vol. 10, no. 1, pp. 16-25, Jan./Feb. 2004

[4] S. Chen, C. Chen, and J. Shih, "Current programmed pixel structures for OLED," in Asia Display/IDW'01, 2001, pp. 400-402.

[5] Y.-C. Lin and H.-P. D. Shieh, "A novel current memory circuit for AMOLEDs," IEEE Trans. Electron Devices, vol. 51, no. 6, pp. 1037-1040, Jun. 2004.

[6] G. B. Levy et al., "An $852 \times 600$ pixel OLED-on-Silicon color microdisplay using CMOS subthreshold- voltage-scaling current drivers," IEEE J. Solid-State Circuits, vol. 37, no. 12, pp. 1879-1889, Dec. 2002.

[7] X. Guo and S. R. P. Silva, "Circuit simulation of current-modulated field emission display pixel driver based on carbon nanotubes," Electron. Lett., vol. 40, pp. 1113-1115, Sep. 2004.

[8] W. Yu-Wu et al., "A novel pixel design for driving CNT-FED display," in Proc. IVMC, 2002.

[9] S.-H. Joon, W.-J. Nam, and M.-K. Han, "A new voltage-modulated AMOLED pixel design compensating for variation in poly-Si TFTs," IEEE Electron Device Lett., vol. 25, no. 1, pp. 690-692, Jan. 2005.

[10] SmartSpice Users Manual, Silvaco International, Santa Clara, CA, 2000.

[11] D. Vallancourt and S. J. Daubert, "Applications of current-copier circuits," in Analog IC Design: The Current Mode Approach, C. Toumazou, F. Lidgey, and D. J. Haigh, Eds. Stevenage, U.K.: Peregrinus, 1990, pp. 515-534.

[12] D. Johns and K. Martin, Analog Integrated Circuit Design. New York: Wiley, 1997.

[13] C. Eichenberger and W. Guggenbuhl, "On charge injection in analog MOS switches and dummy switch compensation techniques," IEEE Trans. Circuits Syst., vol. 37, no. 2, pp. 256-264, Feb. 1997.

[14] NJJ. M. Rabaey, Digital Integrated Circuits: A Design Perspective. Englewood Cliffs: Prentice-Hall, 1996.

[15] D. Engelsen and K. Kortekaas, "Hopping-Electron transport in a fieldemission display," Inf. Display, vol. 20, pp. 22-26, 2004.

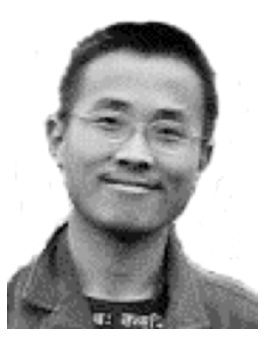

Xiaojun Guo (S'05) was born in Jiangsu, China, in 1981. He received the B.E. degree in electronic materials and devices from Jilin University, Changchun, China, in 2002. He is currently pursuing the Ph.D. degree in the Nano-Electronics Center, Advanced Technology Institute, University of Surrey, Surrey, U.K.

He spent one year doing research on VLSI CAD and CMOS IC design in the Department of Electronic Engineering, Tsinghua University, Beijing, China. His current research focuses on carbon nanotube based devices, poly-Si TFT and circuits for system-on-panel applications.

Mr. Guo is a student member of the IEEE Electron Devices Society, the IEEE Solid-State Circuits Society, and the Society for Information Display (SID). 


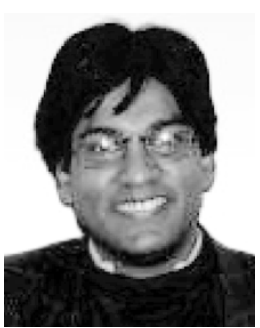

S. R. P. Silva received the Ph.D. degree from the University of Cambridge, Cambridge, U.K.

Currently, he is the Director of the Advanced Technology Institute, University of Surrey, Surrey, U.K., and leads the Nano-Electronics Center. His research interest encompasses a wide range of activities in the areas of nano-electronics and large-area electronics. He has published over 150 papers in refereed journals and over 200 papers presented at international conferences.

Dr. Silva is a fellow of the Institute of Physics and the Institute of Electrical Engineers. In 2002, Prof. Silva was awarded the Charles Vernon Boys Medal by the Institute of Physics, and in 2003 he was awarded the Achievement Award by the Institute of Electrical Engineers. In 2003, he was also awarded the Albert Einstein Silver Medal and Javed Husain Prize by UNESCO for contributions to electronic devices. He holds the Chair in Solid State Electronics at the University of Surrey. 